\title{
\begin{tabular}{|l|l|l|l|l|}
\hline $\mathrm{M}$ & $\mathrm{R}$ & $\mathrm{S}$ & Internet Journal of & Nitride Semiconductor Research \\
\hline
\end{tabular}
}

Volume 1, Article 26

\section{MOCVD Equipment for Recent Developments towards the Blue and Green Solid State Laser}

\author{
H. Jürgensen, D. Schmitz, G. Strauch, E. Woelk \\ AIXTRON GmbH \\ M. Dauelsberg, L. Kadinski, Yu. N. Makarov \\ Lehrstuhl für Strömungsmechanik, University of Erlangen-Nürnberg
}

This article was received on June 2, 1996 and accepted on October 26, 1996.

\begin{abstract}
For the growth of an electrically pumped lasing nitride emitter, the development of the MOCVD equipment and the process are mutually dependent. Most important is the implementation of the rapid temperature changes that are required between the growth of the different layers of a device structure. Equally important is to provide a reaction chamber that develops a stable gas phase at all growth temperatures used in the process. In this paper we will give insight in the technology and the relationship between processes and equipment. The development of the reation chamber was supported by mathematical modeling that formed the basis for the selection of appropriate process parameters for growth of group-III nitrides. The modeling consists of the numerical solution of the Navier-Stokes equations coupled with heat transfer and mass transport of the chemical species. The modeling of radiative heat transfer takes into account the effect of changing surface radiative properties. These changes result from the coating of the reactor inner surfaces during the growth run. Coupled flow dynamics and chemistry including homogeneous and heterogeneous reactions play an important role for predicting growth rate distributions on the susceptor area. At the practically used high temperatures, group-III metalorganics turn out to be almost entirely decomposed and it is the mass transport of these decomposition products to the growing layer that is assumed to control the growth rate in accordance with experimental observations.
\end{abstract} AIXTRON GmbH,

\section{Introduction}

In the quest for blue solid state light emitters, the efforts have shifted towards the research on group III-nitrides semiconductors. Most of the layers used today for the commercial blue and green LED - the device that caused the dramatic change of focus during the last two years - are being produced by metal organic chemical vapor deposition (MOCVD). For the growth of an electrically pumped laser based on III-N materials the development of the equipment and the process is strongly depending on the capabilities of the equipment. Most important is the implementation of the rapid temperature changes that are required for the growth initiation cycle, for temperature changes between the growth of the different layers of a device structure and for the in-situ generation of $p$-type doping. Equally important is a reaction chamber that develops a stable gas phase at all growth temperatures, pressures and flows that are used in the process.

\section{MOCVD Reactor Design}

The MOCVD of GaN and its relatives requires process temperatures that are well above those that are usually encountered in the well established MOCVD of GaAs and its relatives. To enable these temperatures and at the same time maintain the uniformity and purity of the material layers formed in the process, a considerable amount of modifications to the then existing technology was required. As a starting point for our development we decided to use the Planetary Reactor" [1]. Figure 1 shows a cross section of the reactor with a wafer capacity of $7 \times 2$ " wafers. An inductive heater brings the susceptor to a maximum temperature of $1600^{\circ} \mathrm{C}$. Due to the small thermal mass of the 
susceptor, very fast heat-up and cool-down cycles with a speed of up to $6^{\circ} \mathrm{C} / \mathrm{sec}$ can be achieved. For the growth of device structures, multiple temperature changes are required during a growth run and the employment of fast temperature changes between the growth steps is necessary.

The two flow concept which has been used on the Planetary Reactor" prevents the pre-reaction between the metalorganic compounds and the hydrides [2]. The reagents are separated into two carrier gas flows that combine at the injector shortly upstream of the substrate. The thermal management of the reactor in particular is a very critical parameter set. To preserve less stable compounds as trimethyl indium, the injection zone is kept at temperatures below $300^{\circ} \mathrm{C}$. Prior to the design and the construction of the reactor, simulations showed that the gas phase in the reactor will be stable and laminar at the process conditions [3]. This finding was verified in actual growth runs. The prediction of the flow, concentrations and the temperatures in the reactor consists of the numerical solution of the Navier-Stokes equations coupled with heat transfer and mass transport of key chemical species. The modeling of radiative heat transfer employs multi-band gray-diffusive radiation exchange between solid boundaries in the reactor and takes into account the effect of changing surface radiative properties due to formation of coatings on the various surfaces in the reactor [4] [5]. Accurate heat transfer calculations are critical, because precursor decomposition and formation of deposits are determined by the temperature distribution in an MOCVD reactor. Coupled flow dynamics and chemistry including homogeneous and heterogeneous reactions play an important role for predicting growth rate distributions on the susceptor during growth of III-nitrides. At the high temperatures, group-III metalorganics turn out to be almost entirely decomposed an it is the mass transport of the decomposition products to the growing layer that is assumed to control the growth rate. This assumption allows to explain the experimental observations.

The growth temperatures are adjusted with a precision of $0.1^{\circ} \mathrm{C}$ between 450 and $1050^{\circ} \mathrm{C}$. The precise temperature control of the quartz ceiling (wall adjacent to the susceptor) inside the reactor is used to keep the only reactor wall at a favorable temperature for the process $\left(300\right.$ to $\left.600^{\circ} \mathrm{C}\right)$. The controlled temperature allows to minimize deposits [6]. The specially developed growth initiation cycle was used to form a thin GaN layer on the substrates which were pretreated with a nitridation process. The total flow rates and the gas flow the two-flow inlet are used to optimize both growth rates and uniformity while growth rates and V/III ratio can be adjusted independently.

\section{Theory and Modeling of the Process}

Flow, heat transfer and the chemical species mass transport in the reactor are governed by conservation equation for total mass, momentum and energy, supplemented by the equation of state and the convective and diffusive transport of the chemical species. The model also includes homogeneous and heterogeneous chemical reactions. The radiative heat transfer model comprises diffuse surface radiation exchange between the solid boundaries of the reactor (walls) with a non-participating medium and the semi-transparent quartz wall (ceiling). The wavelength dependence of the optical properties is also included in the model. At the ceiling the temperature is determined by conjugate heat transfer including convective cooling by the variable gas mixture $\left(\mathrm{N}_{2}\right.$ and $\left.\mathrm{H}_{2}\right)$ that couples this wall to a heat sink separated from the wall by a gap of $1 \mathrm{~mm}$. the chemical modeling includes the relevant gas phase decomposition steps of all precursors and the surface chemistry of the growing GaN. A complete description of the entire model and calculations is given elsewhere [5]. The model is the most comprehensive computer simulation that has been used for the first time to model the MOCVD reactor and the process.

In the following the figures are discussed that show isoline plots of some selected gas species and streamlines. The simulations also yielded the temperature distribution in the gas phase and the concentration distribution of other species that are reported here. The concentration isolines for $\mathrm{NH}_{3}$ and $\mathrm{N}_{2}$ show that there is a very efficient surface catalytic conversion of $\mathrm{NH}_{3}$ to $\mathrm{N}_{2}$ at the $\mathrm{GaN}$ surface, whereas no such reaction takes place on uncoated graphite of the susceptor. This comparison is chosen as it proves that low pressure conditions result in advantageous condition if the proper adjustment of gas flows and carrier gas is understood.

Figure 2 and Figure 3 show the streamlines and $\mathrm{NH}_{3}$ concentrations inside the reactor at 1000 mbar with $\mathrm{H}_{2}$ as a carrier gas (Figure 2) and at 100 mbar with $\mathrm{N}_{2}$ as a carrier gas (Figure 3). Under both conditions the $\mathrm{NH}_{3}$ is cracked at different speeds. The decomposition is supported by the fact that in this radial flow reactor all gases are decelerated in the radial flow direction. This allows to use much lower $\mathrm{NH}_{3}$ flow rates and $\mathrm{V} / \mathrm{Il}$ flow ratios.

The total pressure reduction leads to increased gas velocities, but no significant changes in growth rates are observed nor predicted, because pressure dependencies cancel out each other in the convective and diffusive terms. This is shown for $\mathrm{H}_{2}$ in Figure 4, where the $\mathrm{GaN}$ growth rate is plotted in radial flow direction from the reactor center. From the leading edge of the wafer to trailing edge the growth rate drops almost linearly. The rotation of the 
wafers under such a growth rate profile yields a uniformity of $\pm 1 \%$ to $\pm 1.5 \%$. The use of $\mathrm{N}_{2}$ as a carrier gas has many practical advantages in contrast to the use of hydrogen. Firstly, as shown in Figure 3, the $\mathrm{N}_{2}$ concentration at the growing surface does not change significantly leading to a higher lateral uniformity of the process conditions. For the process using $\mathrm{H}_{2}$ as a carrier gas, the concentration of $\mathrm{N}_{2}$ originating from the cracking of the $\mathrm{NH}_{3}$ varies

significantly in the growth zone. Secondly, the use of nitrogen as a carrier gas is safer and cheaper than the use of hydrogen. Nitrogen has been successfully used as a carrier gas in the growth of other III-V semiconductor films [7]. In the following all reported results were obtained using nitrogen as a carrier gas at $100 \mathrm{mbar}$.

To understand the implications of the differences between the use of hydrogen and nitrogen as a carrier gas, we have to look a the properties of both gases and their relevance for the process. The density of $\mathrm{N}_{2}$ is higher, the dynamic viscosity, the heat conductivity and the specific heat capacity are lower than for $\mathrm{H}_{2}$. Thus, using $\mathrm{N}_{2}$ results in higher Reynolds numbers. To obtain the same Reynolds numbers when using hydrogen, the flow velocity had to be much higher and therefore the consumption of hydrogen gas would go up. Vice versa, if a process for hydrogen exists, the change to nitrogen results in a decrease of the flow rate. This effect is used for the GaN deposition here, by decreasing the total flow rate from initially approx. $20 \mathrm{l} / \mathrm{min}$ hydrogen to $10 \mathrm{l} / \mathrm{min}$ nitrogen (solid line in Figure 5) and $2.5 \mathrm{l} / \mathrm{min}$ nitrogen (dashed line in Figure 5). At $10 \mathrm{l} / \mathrm{min}$ the growth rate maximum is still blown far into the reactor so that the wafer edges show a lower growth rate than the rest of the wafer. At $2.5 \mathrm{l} / \mathrm{min}$ the uniformity is improved to less than $\pm 1 \%$. Total growth rate and uniformity can be varied in between these values by changing the ratio of the two flows entering the reactor.

\section{Growth Results of III-N Materials}

All layers were grown on basal plane sapphire substrates. epitaxial growth of GaN layers was typically carried out at a temperature of $1050^{\circ} \mathrm{C}$ and a total pressure of $100 \mathrm{mbar}$. To obtain high uniformity layers, the individual wafers were rotated at a speed of about $50 \mathrm{rpm}$. Ammonia gas, trimethyl gallium, trimethyl indium, and trimethyl aluminum are used to grow the crystalline alloy films. In most of the growth runs, nitrogen was used as a carrier gas. The growth of a contiguous layer requires a nucleation layer grown at low temperatures and a proceeding nitridation process.

The typical layer shown in the following is between 1.0 and $2.0 \mu \mathrm{m}$ thick and was obtained after 1 hour of growth. Higher growth rates can be obtained by adjusting a higher molar flow of the group-III precursors in the reactor. The typical background carrier concentration of GaN was determined by Hall measurement and was found to be $10^{17} \mathrm{~cm}$ -3 and a carrier mobility of $250 \mathrm{~cm}^{2} / \mathrm{Vs}$. Structural quality of the material as determined by $\mathrm{x}$-ray diffraction improved using an advanced nitridation-nucleation-anneealing procedure. The narrowest line width of the x-ray rocking curve was around 35 arcsec. However, the most interesting properties of III-N films for device production are the optical data and their distribution over large substrate areas.

The topographic evaluation of optical properties of a single, undoped GaN layer on a 2" wafer by photoluminescence (PL) is shown in the following figures. The distribution of the emission wavelength in Figure 6 shows a standard deviation of $0.42 \mathrm{~nm}$. The emission wavelength at maximum intensity shows a rotational symmetry. The band gap appears to be higher in the center of the wafer than on the edge. Since in a binary material there should not be any wavelength difference at all, and the thickness of the layer was very uniform across the wafer, we suspect that strain in layer causes the shift in wavelength. The radial symmetry could also be explained by a slight radial temperature variation of the $\mathrm{Al}_{2} \mathrm{O}_{3}$ substrate which in turn could be caused by uncontrolled thermal coupling to the susceptor. The corresponding luminescence line width in Figure 7 reflects the uniformity of various parameters such as the perfection of the initial growth states over the substrate area (nitridation, nucleation). The average value at room temperature is $4.89 \mathrm{~nm}$ corresponding to $44 \mathrm{meV}$. A standard deviation of $0.33 \mathrm{~nm}$ measured over a 2" wafer represents the outstanding uniformity of the reactor parameters. A similar picture is given by the topography of the luminescence intensity of the same layer depicted in Figure 8.

The film thickness distribution of the layer as measured by reflectivity measurement is given in Figure 9. A pronounced circular structure again reflects the rotational symmetry of the layer properties. The film thickness distribution is correctly reflected whereas the absolute value is in arbitrary units not converted to real dimensions.

Finally the distribution of sheet resistivity shows a mean deviation of $1.85 \%$. The sheet resistivity stands for the background carrier concentration and the uniformity of growth condition influencing the incorporation of donors. A contour mapping of the sheet resistivity of the layer is given in Figure 10.

The optical properties of ternary alloys are presented by a set of PL measurements in Figure 11 and 12 . These are taken from an AlGaN layer with low Al-Content grown on a standard nucleation/buffer layer. The room temperature $\mathrm{PL}$ wavelength distribution across the 2" wafer represents a uniformity of $0.25 \mathrm{~nm}$ at a center wavelength of $340 \mathrm{~nm}$ 
$(3.65 \mathrm{eV})$, Figure 11. The average line width and its distribution on the wafer area is equal to the binary compound layer (Figure 12). The even uniformity does not allow the resolution of the rotational symmetry at the $1 \mathrm{~nm}$ step size.

\section{Conclusion}

The efforts of device development towards blue light emitting devices has driven a strong demand in MOCVD reactors with the capability to grow the necessary quality and uniformity on large substrate areas. Because of the complexity of the growth chemistry and the extremely narrow process window (temperature) for the growth of suitable quality III-N material films, the production reactor development focused on a more stringent scenario than in the regular III-V material field. The transfer of a proven technology to the new material system GalnAIN required the support of an advanced modeling of the process. The results presented here demonstrate the successful application of numerical modeling and the subsequent verification of the simulations for the growth of nitride material films for advanced blue and green light emitting diodes.

\section{References}

[1] P. M. Frijlink, J. L. Nicolas, H. P. M. M. Ambrosius, R. W. M. Linders, C. Waucquez, J. M. Marchal, J. Cryst. Growth 115, 203 (1991).

[2] C. Chen, unpublished (1996).

[3] M Dauelsberg, L Kandinski, YuN Makarov, E Woelk, G Strauch, D Schmitz, H Jurgensen, Inst. Phys. Conf. Ser. 142, 887 (1996).

[4] L. Kadinski, Yu. N. Makarov, M. Schäfer, V. Yuferev, M. Vasil'ev, J. Cryst. Growth 146, 209 (1994).

[5]F. Durst, L. Kadinski, Yu. N. Makarov, M. Schäfer, V. S. Yuferev, M. G. Vasil'ev, "Advanced Mathematical Models for Simulation of Radiative Heat Transfer in CVD Reactors", presented at $11^{\text {th }}$ Intl. Conf. on Crystal Growth, The Hague, 1995

[6]G. Strauch, J. Hergeth, B. Wachtendorf, M. Volk, E. Woelk, "In-situ Monitoring of Process Parameters and Boundary Conditions", Proc. Intl. Conf. Blue Lasers and LEDs, Chiba, Japan, 1996

[7] H. Hardtdegen, M. Hollfelder, R. Meyer, R. Carius, H. Münder, S. Frohnhoff, D. Szynka, H. Lüth, J. Cryst. Growth 124, 420 (1992).

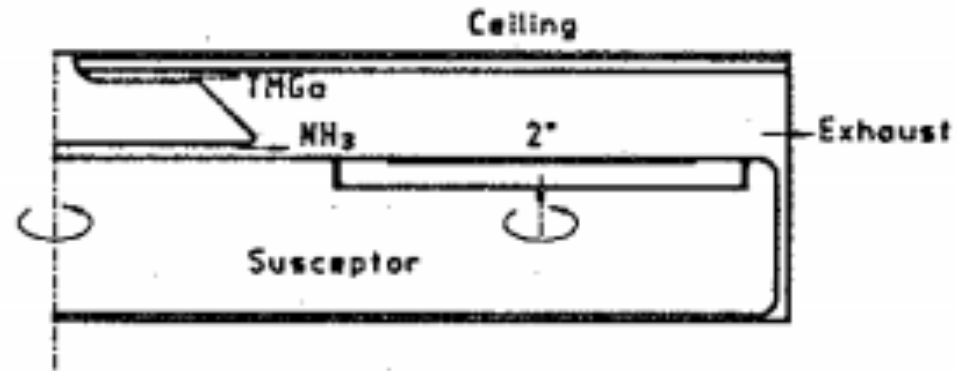

$7 \times 2^{*}$ A $1 \times 2000$

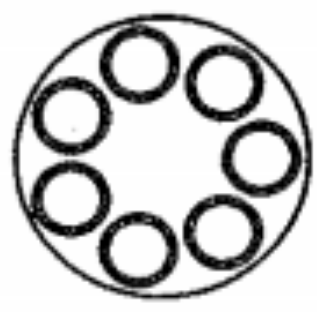

$15 \times 2^{*}$ A $1 \times 2400$

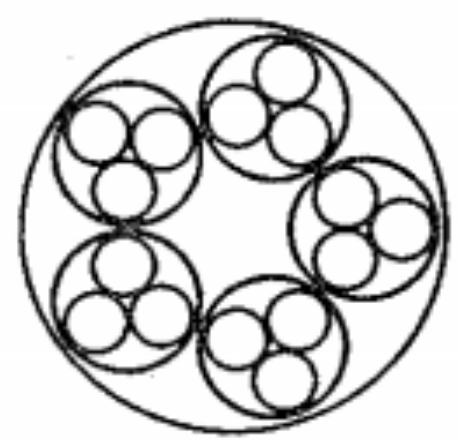

Figure 1. Cross section of Multiwafer Planetary Reactor $^{\circledR}$ for III-N growth 


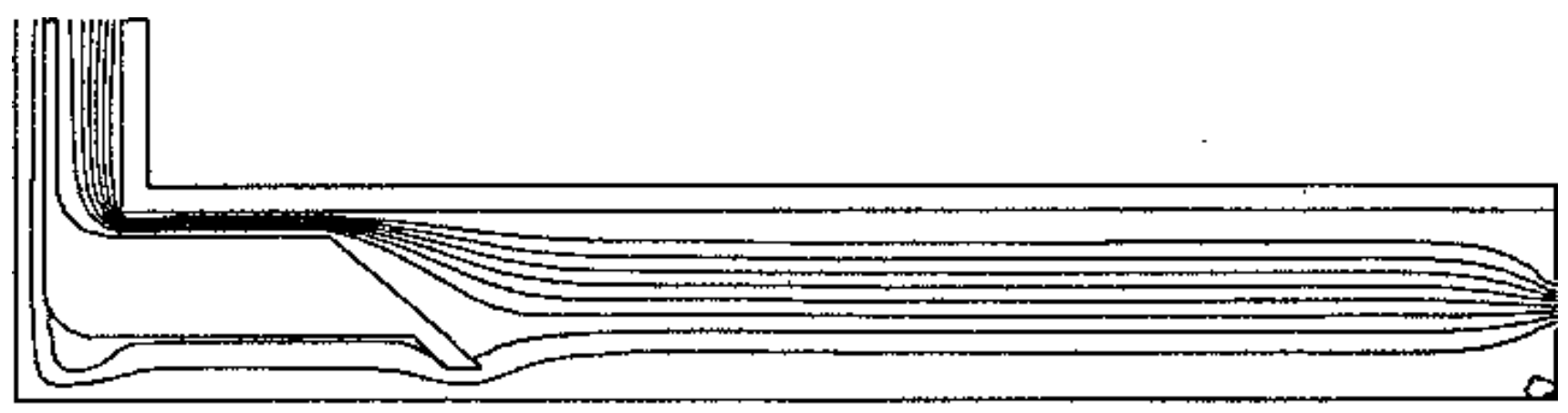

\section{Streamlines}

Figure 2a. Streamlines in the planetary Reactor at 1000 mbar

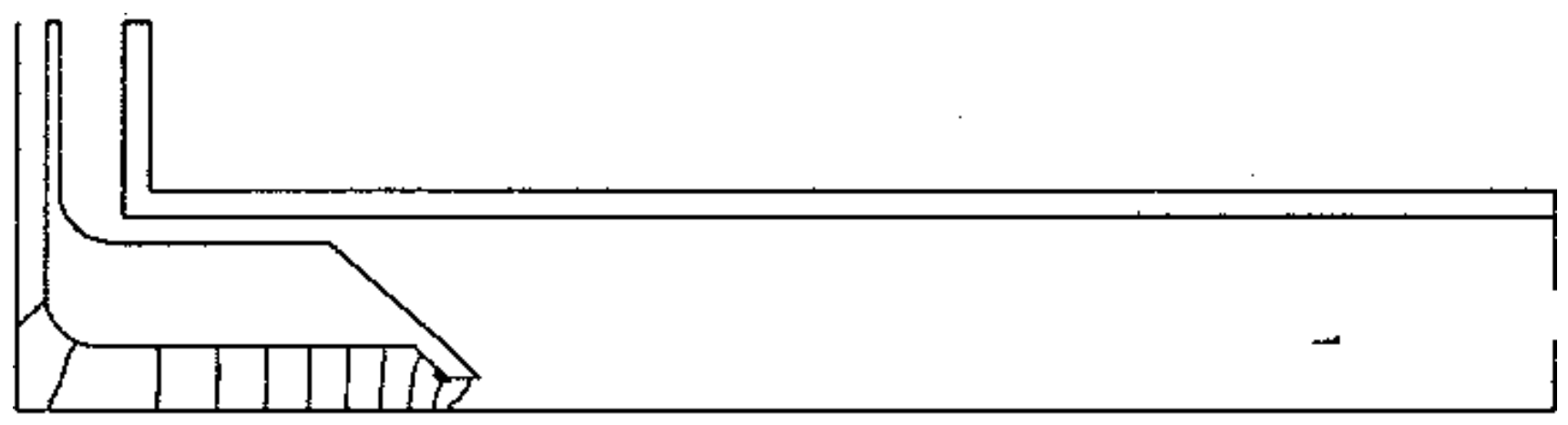

$\mathrm{NH}_{3}$ Concentration

Figure $\mathbf{2 b}$. $\mathrm{NH}_{3}$ concentration profile in the planetary Reactor at 1000 mbar

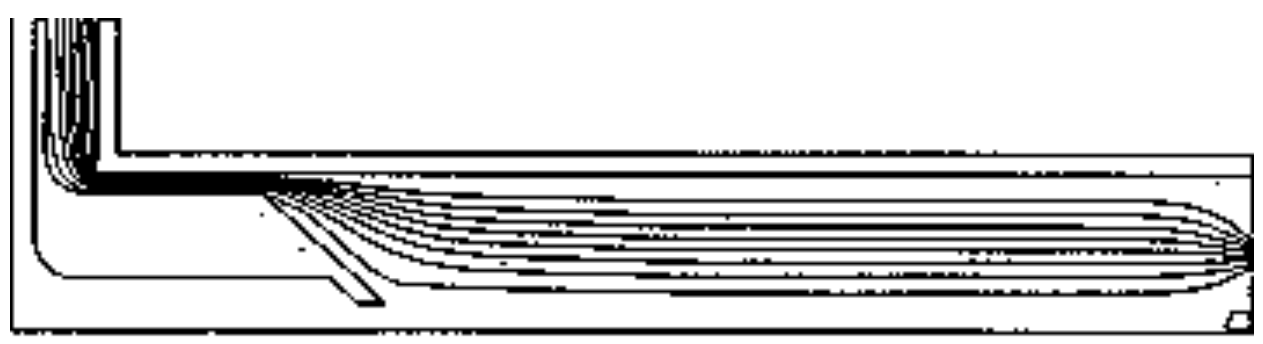

\section{Streamlines}

Figure 3a. Streamlines in the planetary Reactor at $100 \mathrm{mbar}$ 


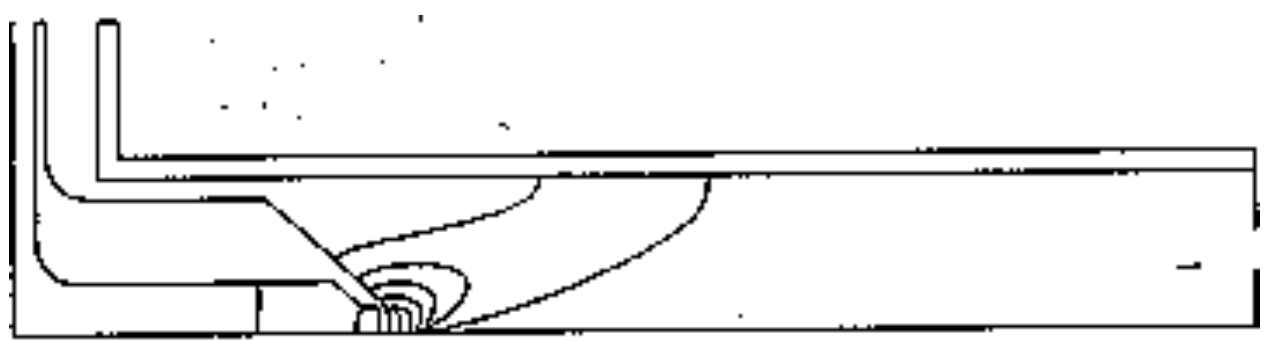

\section{$\mathrm{NH}_{3}$ Concentration}

Figure 3b. $\mathrm{NH}_{3}$ concentration profile in the planetary Reactor at $100 \mathrm{mbar}$
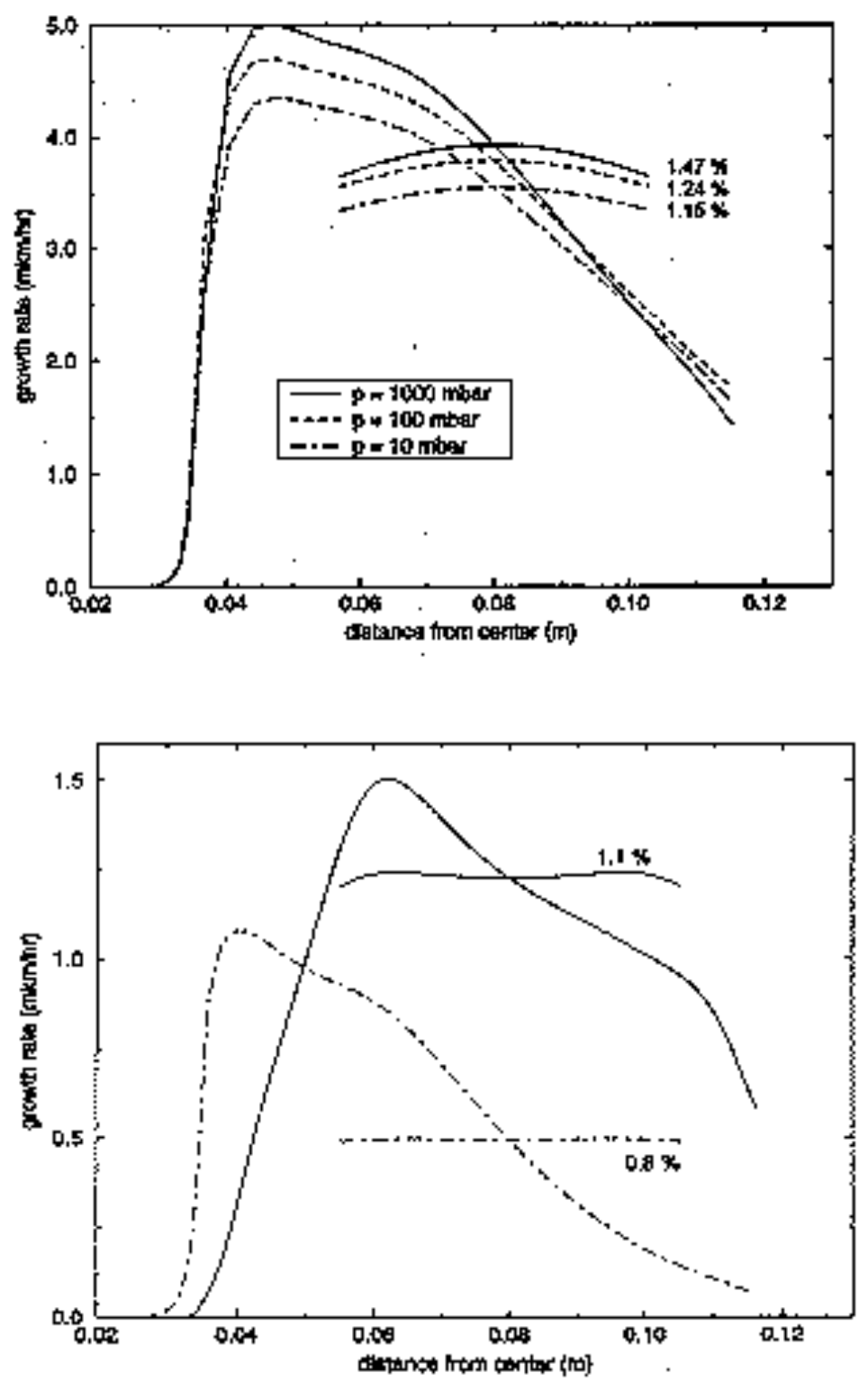

Figure 4. Calculated growth rate distribution in radial direction over susceptor with $\mathrm{H}_{2}$ carrier gas, total flow $20 \mathrm{l} / \mathrm{min}$, TD $1000^{\circ} \mathrm{C}$.
Figure 5. Calculated growth rate distribution in radial direction over susceptor with $\mathrm{N}_{2}$ carrier gas, total flow $10 \mathrm{l} / \mathrm{min}$ and $2.5 \mathrm{l} / \mathrm{min}$ (dashed line), TD $1000^{\circ} \mathrm{C}$. 
$\begin{array}{llll}\text { Avg. Width } & : & 4.89 & \mathrm{~nm} \\ \text { Std Deviation : } & 0.93 & \mathrm{~nm}\end{array}$
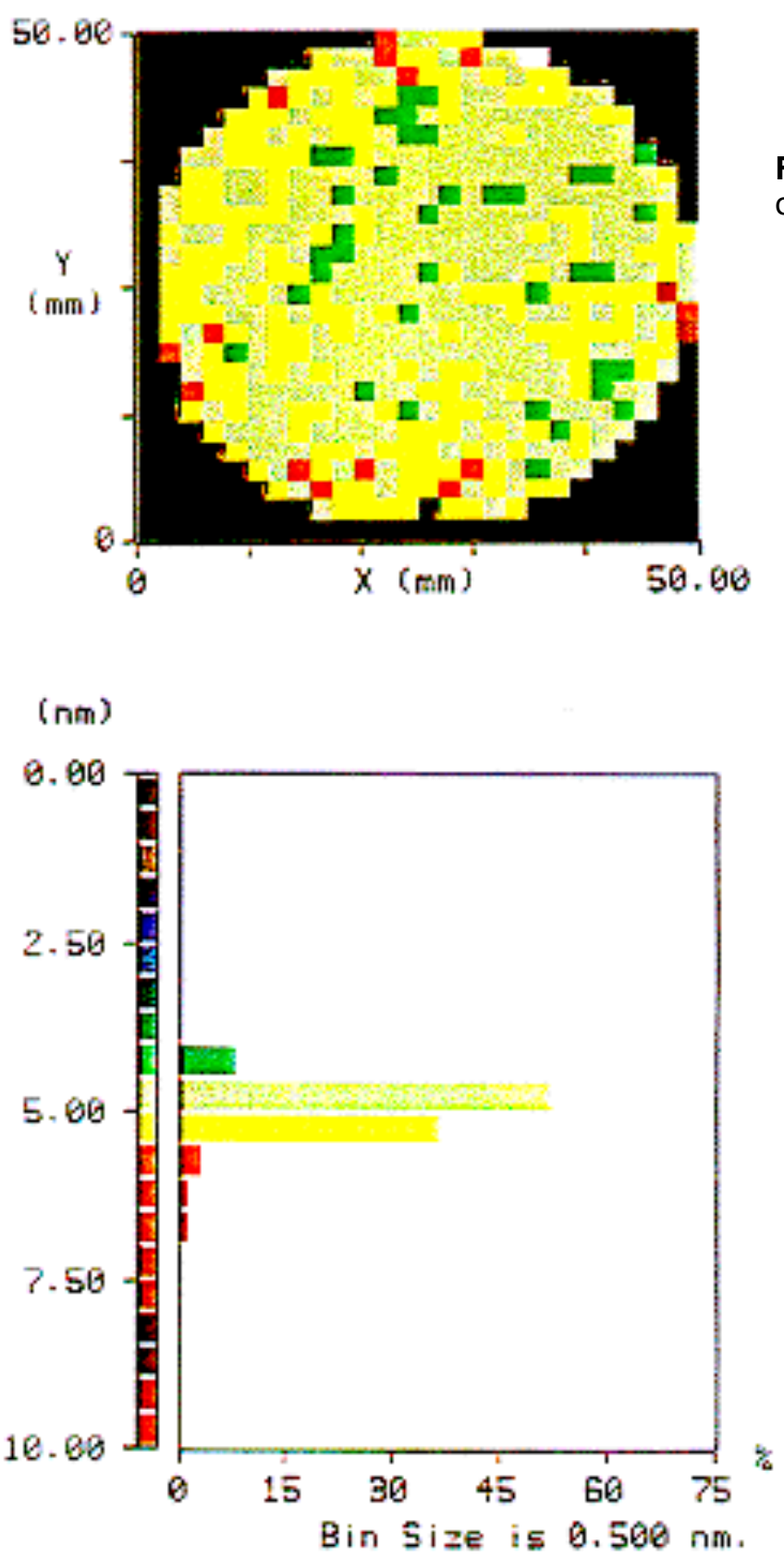

Figure 6. Room temperature PL wavelength topography of GaN layer on 2» wafer
Figure 6a. Color histogram. 
Avg. Wavelensth : $\quad 369.79 \mathrm{~nm}$

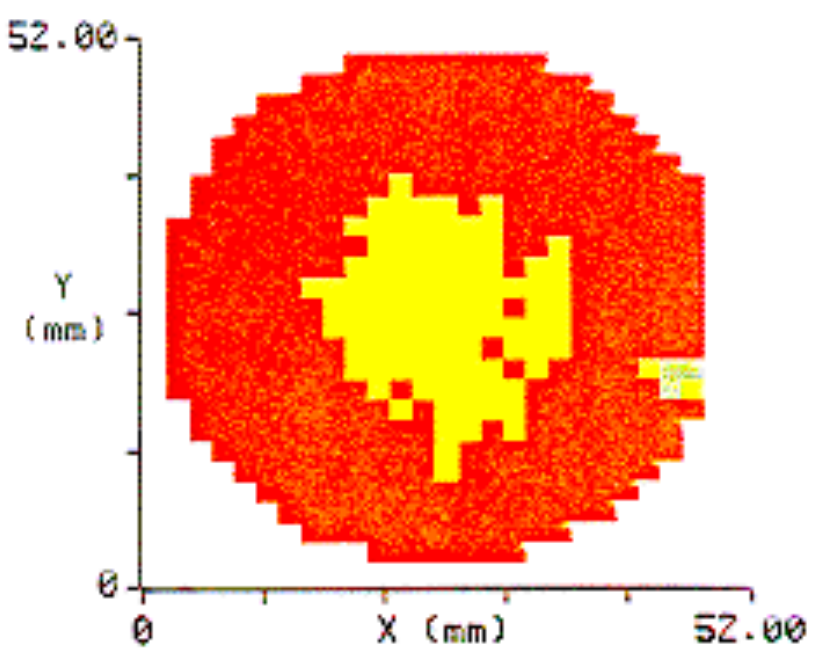

\section{(nm)}

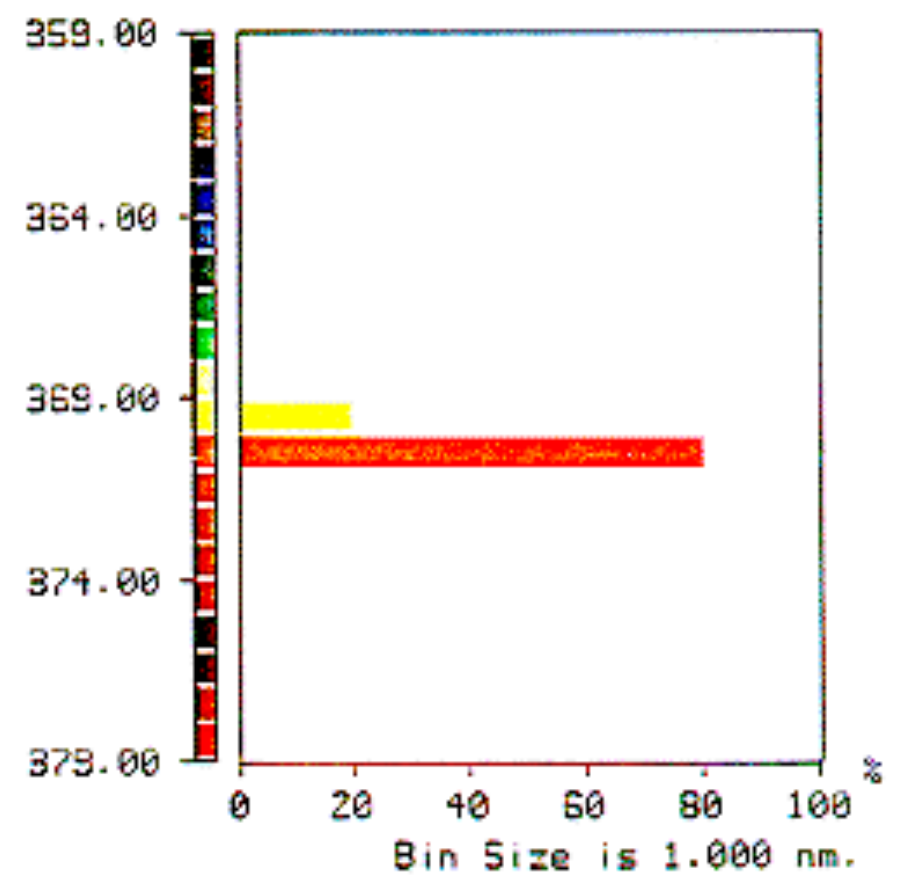

Figure 7. Room temperature PL FWHM topography of GaN layer on 2» wafer
Figure 7a. Color histogram. 


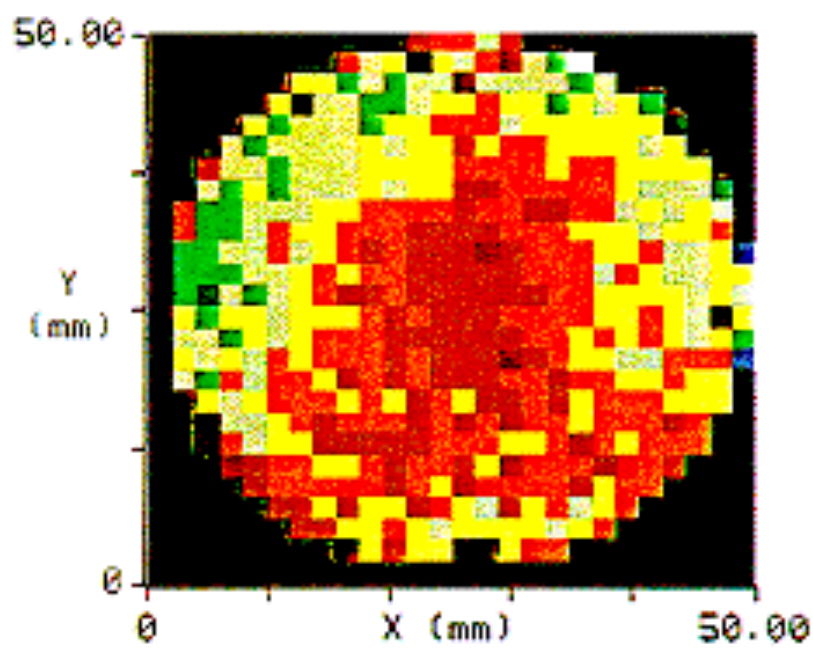

(CU)

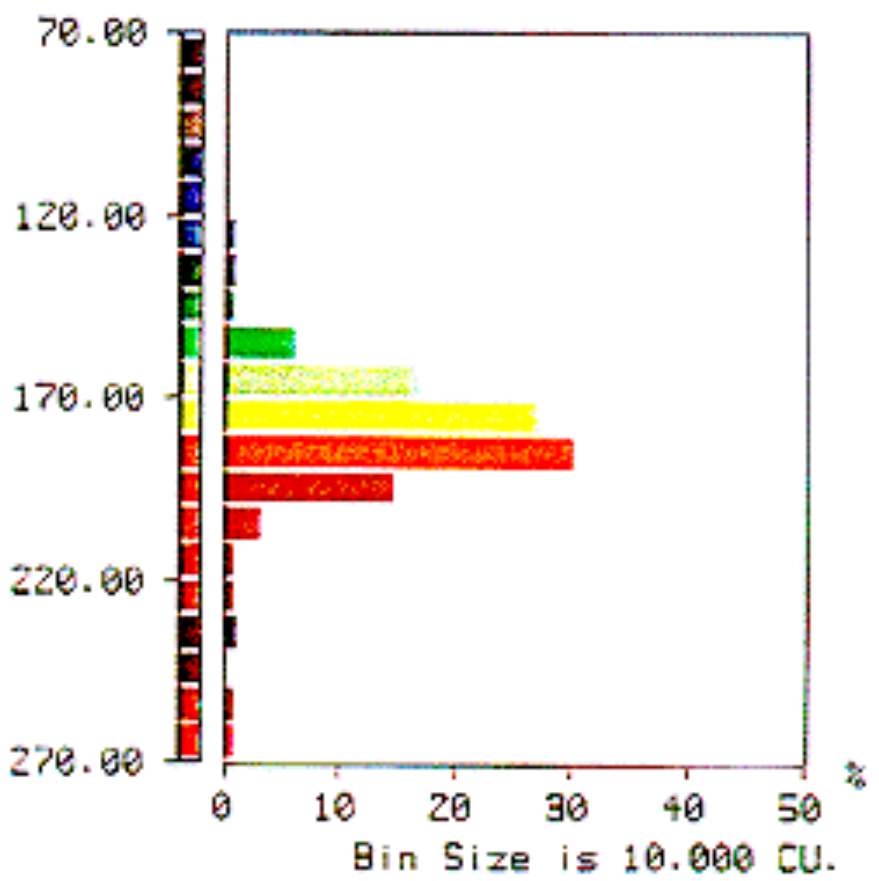

Figure 8. Room temperature PL intensity topography of GaN layer on 2» wafer

Figure 8a. Color histogram. 
Avg. Thickness : $228.36 \mathrm{~nm}$

Std Deviation : $\quad 14.46 \mathrm{~nm}$

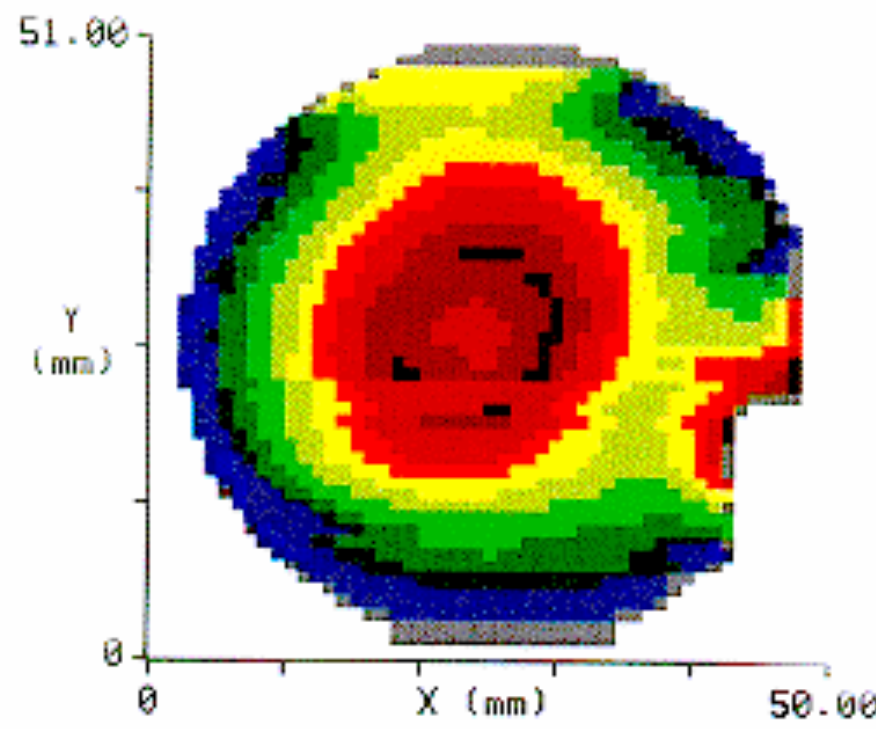

$$
\text { (nin) }
$$

180.00

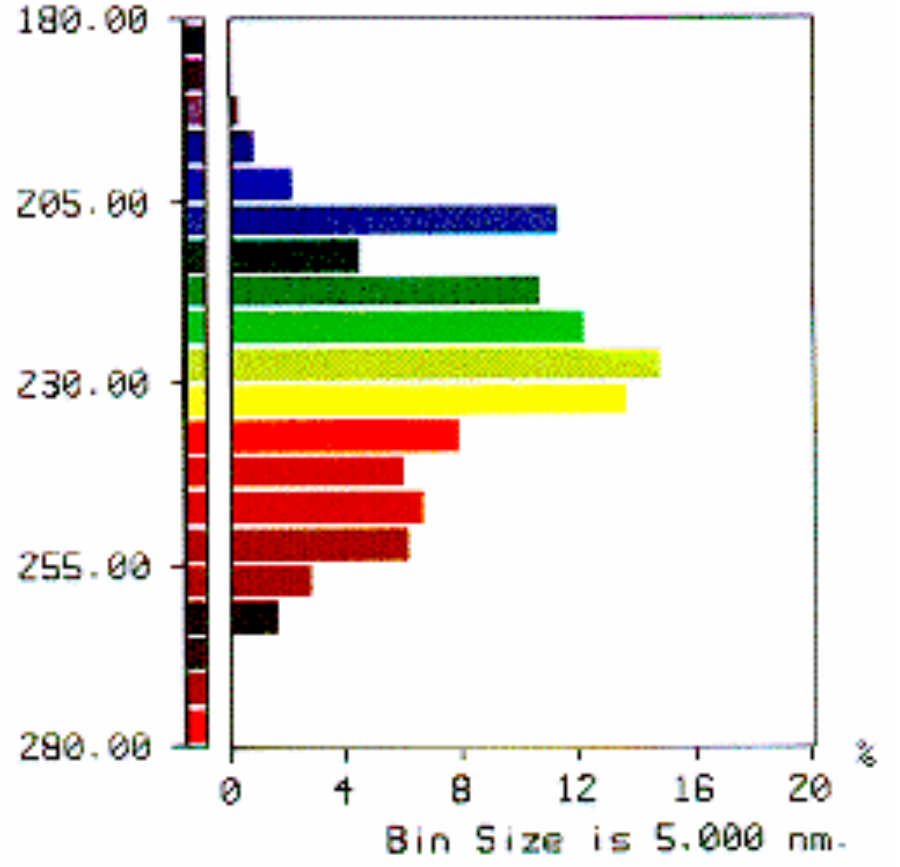

Figure 9. Qualitative film thickness distribution map over 2» GaN layer

Figure 9a. Color histogram. 


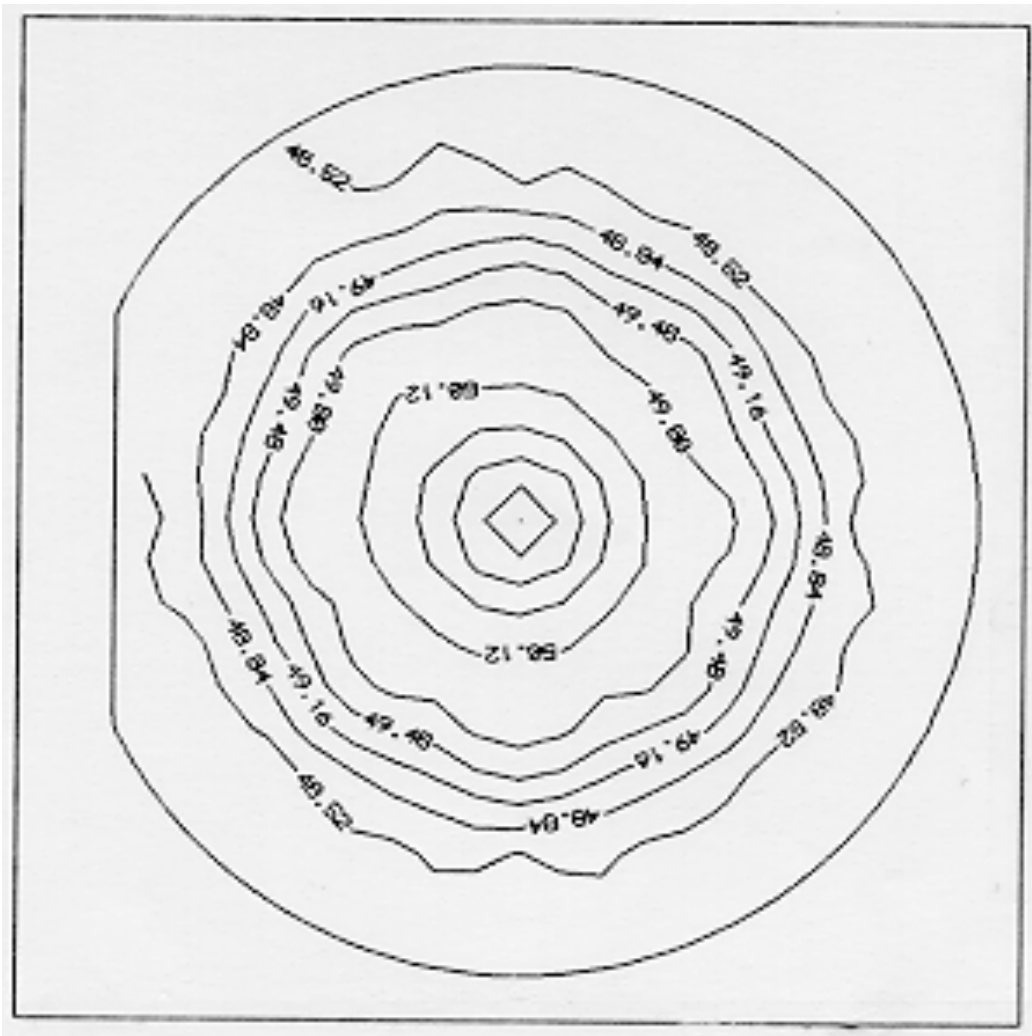

Figure 10. Sheet resistivity map of 2» GaN layer
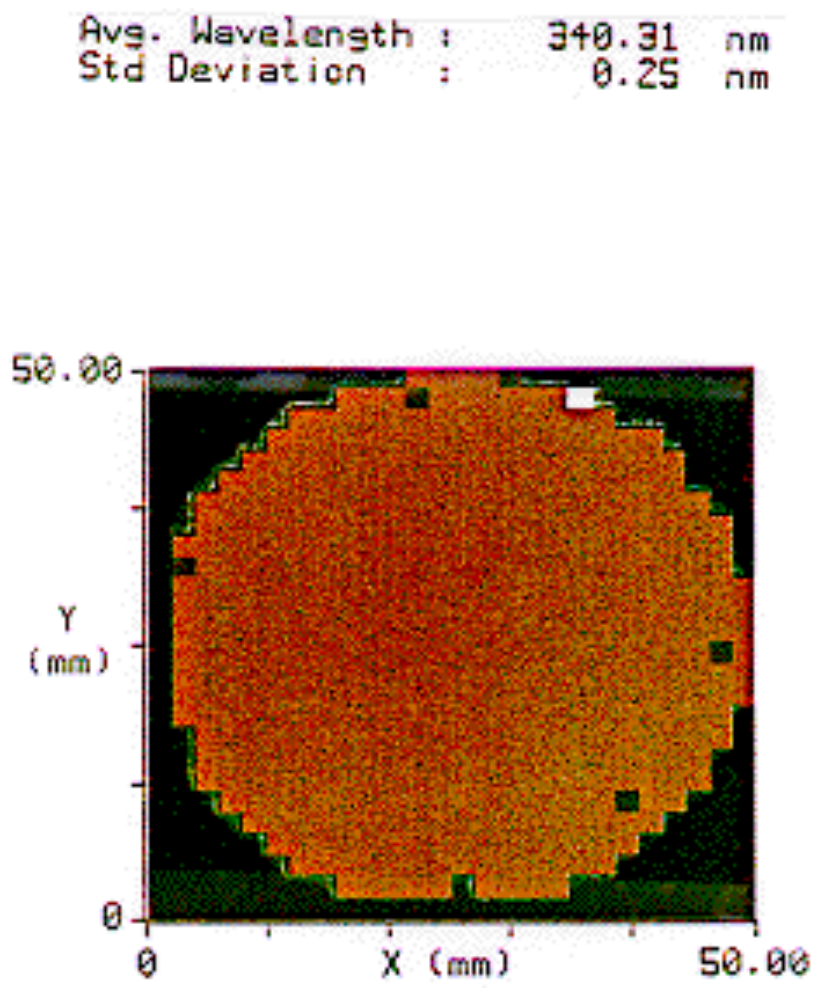

Figure 11. Room temperature PL wavelength topography of AIGaN layer on 2» wafer 
$(\mathrm{nm})$

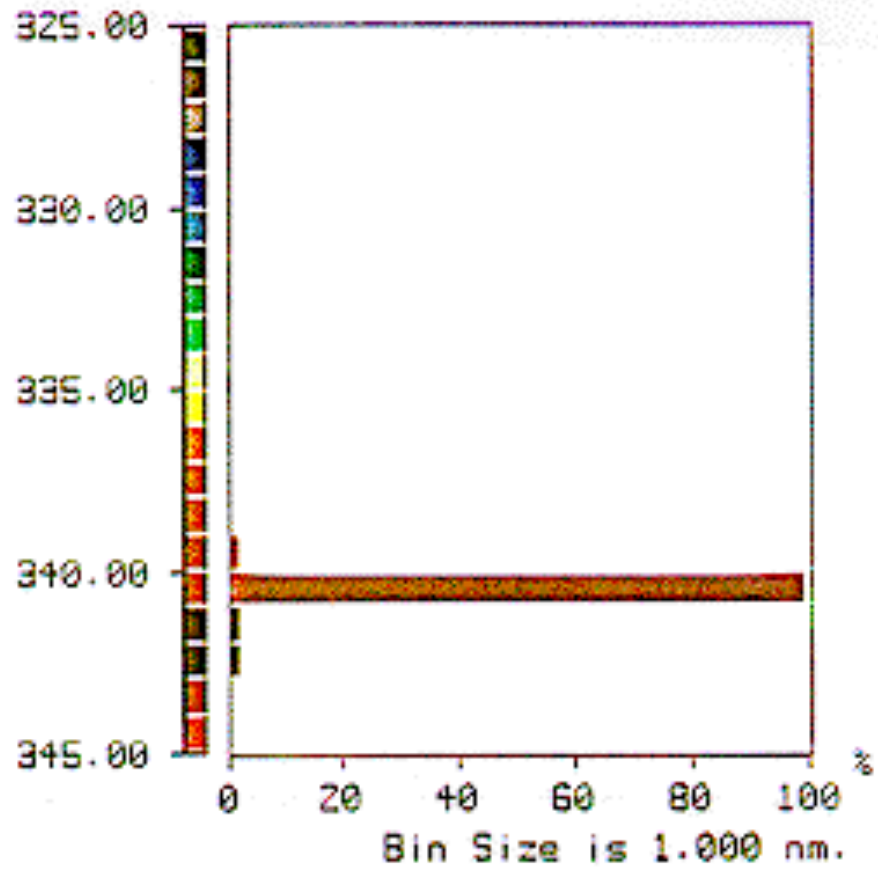

Avg. Width

Std Deviation

$4.89 \mathrm{~nm}$

$0.33 \mathrm{~nm}$

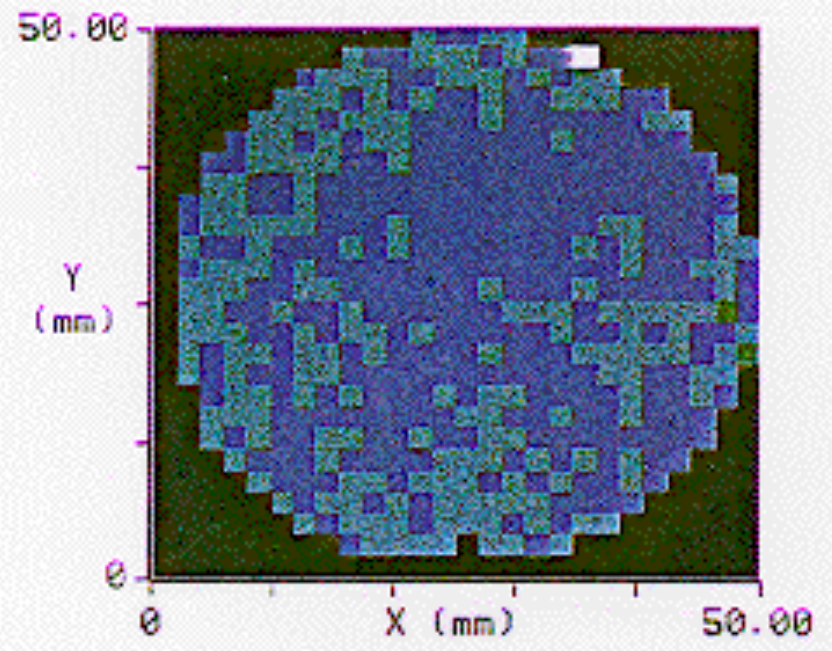

Figure 11a. Color histogram.

Figure 12. Room temperature PL FWHM topography of AlGaN layer on 2» wafer 


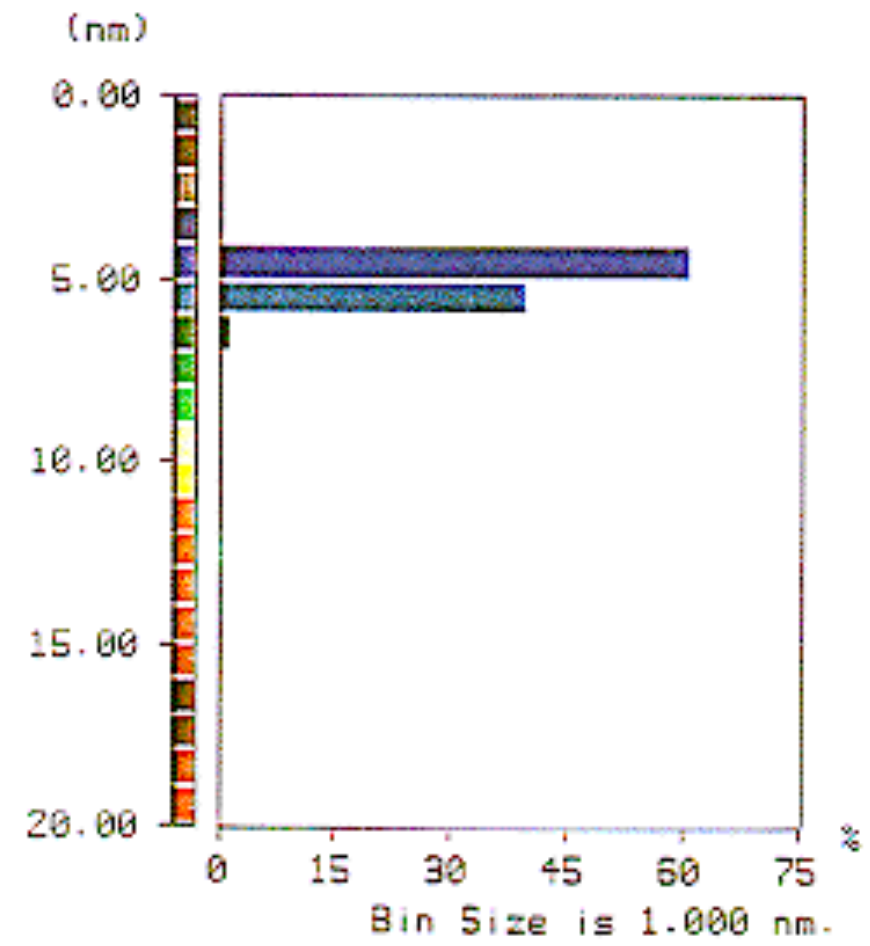

Figure 12a. Color histogram.

(C) 1996-1997 The Materials Research Society

\begin{tabular}{|l|l|l|l|l|}
\hline $\mathrm{M}$ & $\mathrm{R}$ & $\mathrm{S}$ & Internet Journal of & Nitride Semiconductor Research \\
\hline
\end{tabular}

